\title{
Characteristics of the Tooth in the Initial Movement: The Influence of the Restraint Site to the Periodontal Ligament and the Alveolar Bone
}

\author{
Kyoko Shinya ${ }^{1}$, Akikazu Shinya ${ }^{2, *}$, Rizako Nakahara ${ }^{3}$, Yuji Nakasone ${ }^{4}$ and Akiyoshi Shinya ${ }^{5}$
}

\begin{abstract}
${ }^{1}$ Department of Orthodontics, School of Life Dentistry at Tokyo, The Nippon Dental University. 1-9-20 Fujimi, Chiyodaku, Tokyo 102-8158, Japan; ${ }^{2}$ Department of Prosthetic Dentistry and Biomaterials Science, Institute of Dentistry, University of Turku, Lemminkäisenkatu 2, FI-20520 Turku, Finland, and Junior Lecture, Department of Crown and Bridge, School of Life Dentistry at Tokyo, The Nippon Dental University, 1-9-20 Fujimi, Chiyoda-ku, Tokyo 102-8159, Japan; ${ }^{3}$ Department of Orthodontics, School of Life Dentistry at Tokyo, The Nippon Dental University. 1-9-20 Fujimi, Chiyodaku, Tokyo 102-8158, Japan; ${ }^{4}$ Computational Solid Mechanics Laboratory, Department of Mechanical Engineering, Science University of Tokyo. 1-3 Kagurazaka Shinjuku-ku, Tokyo 162-8601, Japan and ${ }^{5}$ Department of Crown and Bridge, School of Life Dentistry at Tokyo, The Nippon Dental University. 1-9-20 Fujimi, Chiyoda-ku, Tokyo 102-8158, Japan
\end{abstract}

\begin{abstract}
It is critical to clarify orthodontic load transfer mechanism from tooth to alveolar bone, and to determine the influence of applied orthodontic force on tooth behaviour. In this study, two dimensional (2-D) finite element (FE) models were constructed to simulate to mechanical behaviour observed during the initial movement of periodontal ligament (PDL) deformation, and to evaluate the effects of the presence of PDL and various restraint sites on tooth behaviour.

A 2-D solid FE model of the tooth-PDL-alveolar bone system was constructed and investigated into stress distribution pattern and displacement. The first analysis was carried out with combinations of FE model with and without PDL. The second analysis was compared with three different sites restraint of alveolar bone.

By incorporating PDL in FE models, excessively large stress values and deformation generated in a tooth and alveolar bone were relieved. Since restraint conditions did not affect a tooth and PDL, but had an effect on alveolar bone, orthodontic force necessary for tooth displacement was transmitted correctly. The results of this study revealed that inclusion of PDL in FE models is indispensable to transmit orthodontic force appropriately when investigating tooth behaviour for orthodontic treatment. Restrained sites affected stress distribution in alveolar bone.
\end{abstract}

\section{INTRODUCTION}

In orthodontic treatment, an esthetics are restored by moving an irregular tooth to right position by applying orthodontic forces to bracket, wire, and elastic band. To displacement of a tooth, large orthodontic force exceeding friction force generated in a bracket and a wire must be applied [1]. The application of large orthodontic force impedes the blood supply of periodontal ligament (PDL), causing ischemia [2-4]. Ischemic situation affects metabolism of oxygen, leading to the initiation of periodontium remodelling processes. Due to strict restrictions on in vivo experiments, orthodontic bone remodelling processes have been mainly analysed from a biomechanical point of view using models simulating what actually occurs in the oral environment. Several studies such as dial gauge method, straight gauge method, distribution method and vibration method have been reported regarding orthodontic tooth movement $[5,6]$. Orthodontic remodelling processes are divided into two stages, i.e. initial tooth movement period when PLD is mainly deformed, and second tooth movement period when a whole periodontium included alveolar bone are deformed

*Address correspondence to this author at the Department of Prosthetic Dentistry \& Biomaterial Science, Institute of Dentistry, University of Turku, Lemminkäisenkatu 2, FI-20520 Turku, Finland; Tel: +358 2333 81; Fax: +358 2333 8390; E-mails: akikazu_dds@msn.com and akikazu.shinya@utu.fi
[7]. Due to the difficulty of making a model of periodontium included PDL, the results from a conventional study using a model are thought to be inaccurate and differ from what actually occurs in a living body. With the development of computer technology, Finite Element (FE) method has been widely used in dentistry for the evaluation of biomechanical situations [8-13]. It is critical to clarify orthodontic load transfer mechanism from a tooth through PDL to alveolar bone, and to reveal the influence of applied orthodontic force on tooth behaviour, which might be precious guidelines for orthodontic treatment.

The aims of this study were to compare and evaluate the effect of the presence of PDL and various restraint sites, during initial tooth movement of PDL deformation, on tooth behaviour and stress distribution patterns, using FE analysis.

\section{MATERIALS AND METHODOLOGY}

Tooth behaviour with or without PDL under various restraint conditions was observed using different two dimensional (2D) FE models of mandibular central incisor.

\section{FE Model Preparation}

A 2D FE model of the tooth-PDL-alveolar bone system (ellipse-shaped) representing mandibular central incisor was constructed (Fig. 1). 
A 2D model was to be in the middle of both buccolingual and mesiodistal diameters and along the long axis of a crown. A 2D model used in this study assumed to have the following dimensions, crown and root lengths $12.0 \mathrm{~mm}$ each, labiolingual diameter of a crown $8.0 \mathrm{~mm}$ and PDL $0.2 \mathrm{~mm}$. The distance between cervical area and base of mandible was assumed to be $16.0 \mathrm{~mm}$ and labiolingual diameter of alveolar bone $10.0 \mathrm{~mm}$ [14]. Two different 2D FE models were prepared for this study, i.e. the tooth- alveolar bone continuum (abbreviated as model without PDL) and the tooth-PDLalveolar bone continuum (model with PDL). As restraint conditions, alveolar bone was restrained in three different sites, i.e. the base of alveolar bone (base), the base of alveolar bone and the lower one half of labiolingual sides (base + side1/2) and the base of alveolar bone and labiolingual sides (a whole side). Thus, four different kinds of FE models were created, i.e. model A (without PDL and base restrained), model $\mathrm{B}$ (with PDL and base restrained), model $\mathrm{C}$ (with PDL and base + side $1 / 2$ restrained) and model D (with PDL and a whole restrained). Analyses were carried out with combinations of analysis 1 (model A and model B), and analysis 2 (model B, model $\mathrm{C}$ and model $\mathrm{D}$ ).

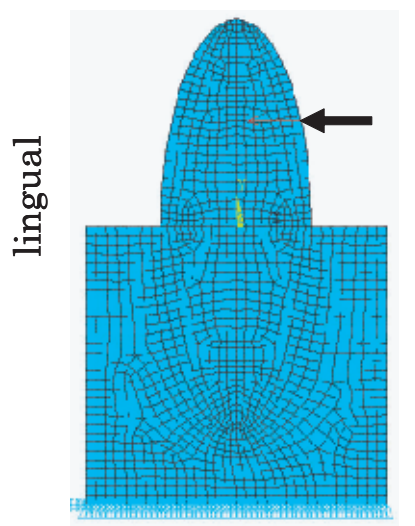

A

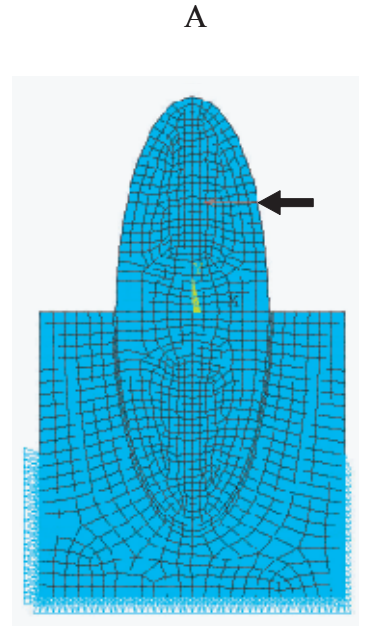

C

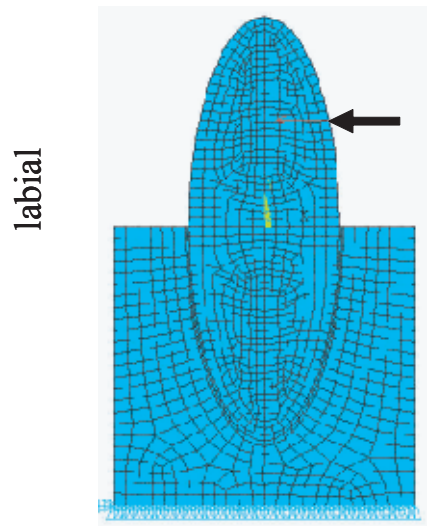

B

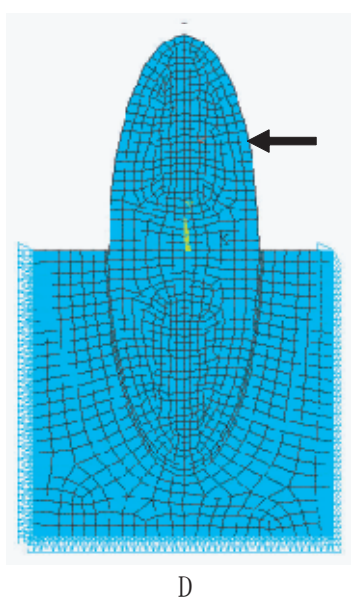

$\triangle$ : restraint site $\leftarrow$ : loading point
Fig. (1). 2-D FE models of mandibular central incisors under various conditions.

A: Model A, without PDL and base restrained, B: Model B, with PDL and base restrained, C: Model C with PDL and base + side 1/2 restrained, D: Model D with PDL and a whole restrained.

\section{Boundary Conditions}

Quadrilateral elements with 8 nodes having middle nodes, consisted of 1,146 elements with 3,575 nodes, were created for this plane analysis. Movements in labiolingual direction on the base of alveolar bone were restrained, which defined as restraint conditions. A single, concentrated load of $1.0 \mathrm{~N}$ [15] was applied from labial to lingual directions at the central part of a crown, where a bracket is to be seated. Analysis was presumed to be linear static. The corresponding material properties such as Young's modulus and Poison's ratio were listed in Table $\mathbf{1}$.

Table 1. Material Properties Used in the Present FEM Simulations

\begin{tabular}{|c|c|c|}
\hline & Young's Modulus (MPa) & Poisson's Ration \\
\hline \hline Tooth Substatance & $8.0 \times 10^{4}$ & 0.30 \\
\hline Periodontal Ligament & $1.8 \times 10^{-1}$ & 0.49 \\
\hline Alveolar Bone & $7.8 \times 10^{3}$ & 0.30 \\
\hline
\end{tabular}

FE model construction and FE analysis were performed on PC workstation (Precision Work Station 530, Dell Computer) using commercially available FE analysis software (ANSYS 9.0 ANSYS Inc. Houston, USA). The width of contour between analyses 1 and 2 was changed so as to differentiate the difference of stress values between them. The stress analysis was performed using equivalent (Von Mises) stress values which summarise the effect of six stress components in a unique value. In the mathematical formula, considering the three principal stress components $(\sigma 1, \sigma 2$ and $\sigma 3)$, the von Mises stress value was defined as follows:

$\sigma \mathrm{VM}=1 / 2 \sqrt{(\sigma 1-\sigma 2) 2+(\sigma 1-\sigma 3) 2+(\sigma 2-\sigma 3) 2}$

\section{RESULTS}

\section{Analysis 1}

The behaviour of mandibular central incisor with or without PDL and base restraint

\subsection{Equivalent Stress Distribution Pattern}

Figs. (2a and 3a) show equivalent stress distribution pattern observed in model $\mathrm{A}$ and model $\mathrm{B}$, respectively. In model $\mathrm{A}, 0.4 \sim 0.8 \mathrm{MPa}$ stresses were found in cervical area and $0.1 \sim 0.4 \mathrm{MPa}$ stresses in alveolar process. Relatively small stresses of $0.1 \sim 0.5 \mathrm{MPa}$ were observed in root apex, showing integrated stress distribution pattern with alveolar bone. Stress distribution pattern demonstrated almost line symmetry against major axis of the tooth.

In model $\mathrm{B}, 0.4 \sim 0.8 \mathrm{MPa}$ stresses were found in cervical area, $0.1 \sim 0.2 \mathrm{MPa}$ stresses in alveolar process, and $0.1 \sim 0.2 \mathrm{MPa}$ stresses in PDL around cervical area. At the corresponding root apex, different magnitude of stresses were observed in PDL $(0.1 \sim 0.3 \mathrm{MPa})$ and in alveolar bone $(0.5 \sim 2.5 \mathrm{MPa})$. Like model A, stress distribution pattern of model $\mathrm{B}$ demonstrated almost line symmetry against major axis of the tooth. 


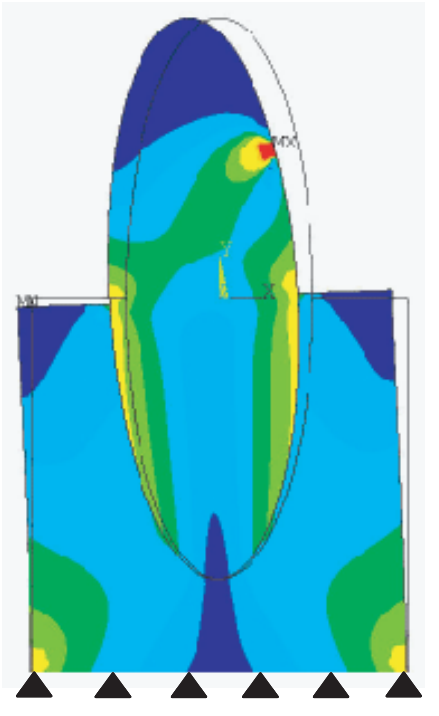

a

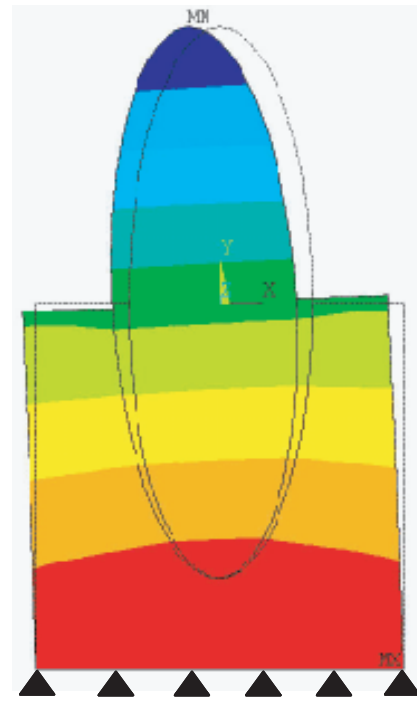

b

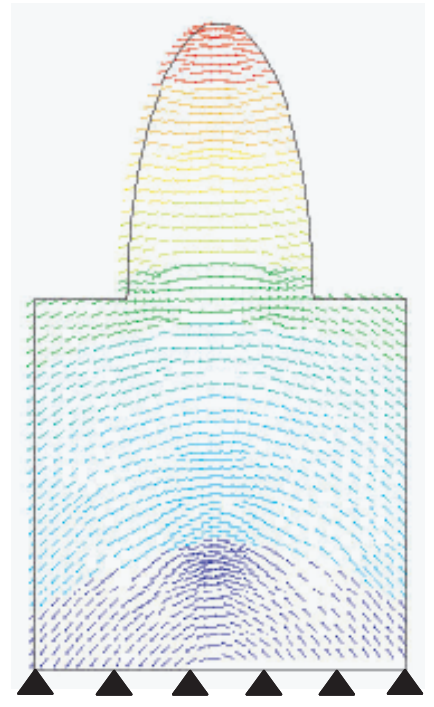

c

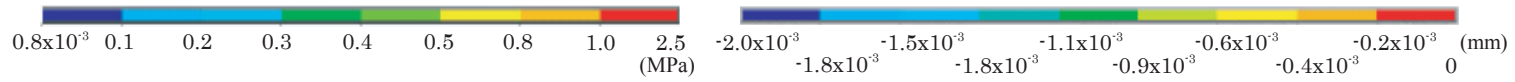

Fig. (2). Results of model A (without PDL and base restrained). a: equivalent (Von Mises) stress distribution, b: displacement, c: vector indication of displacement.

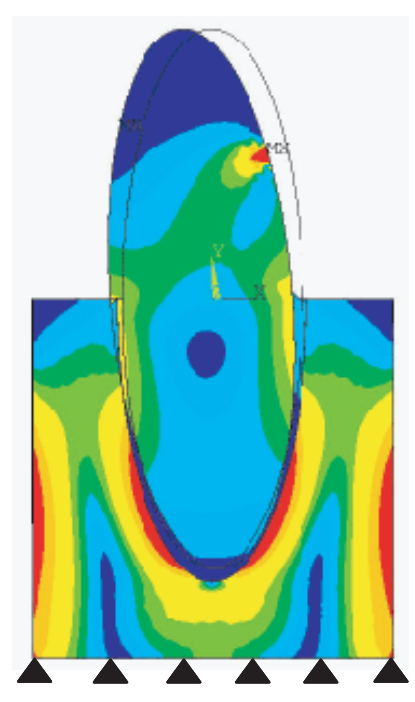

a

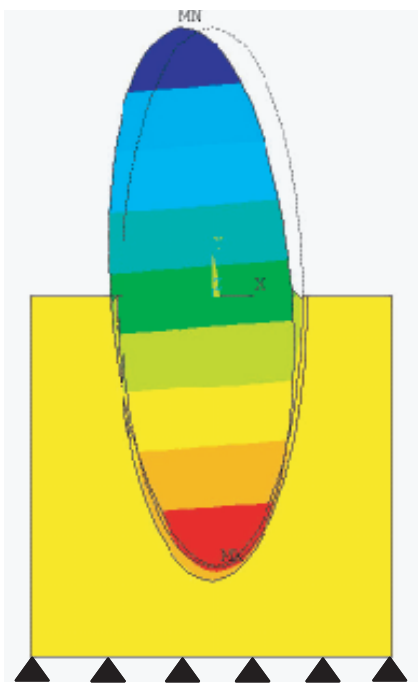

$\mathrm{b}$

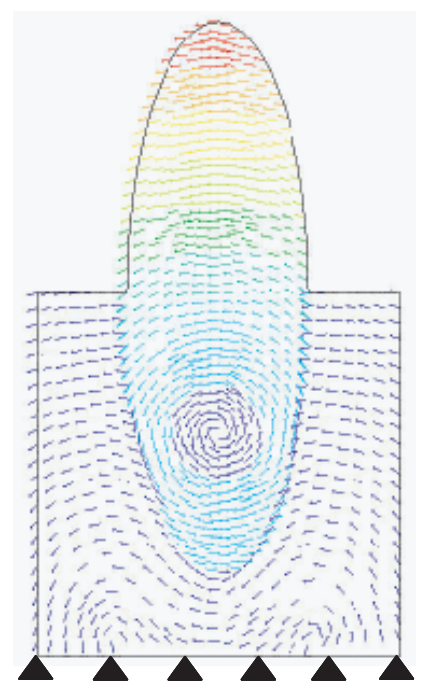

C

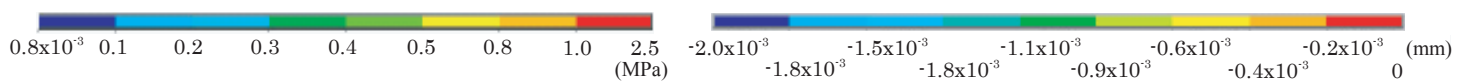

Fig. (3). Results of model B (with PDL and base restrained).

a: equivalent (Von Mises) stress distribution, b: displacement, c: vector indication of displacement.

\subsection{Displacement Distribution Pattern}

An overall displacement of model A (Fig. 2b) was smaller than that of model B (Fig. 3b), and model A was inclined to negative direction of $\mathrm{X}$ axis pivoting on the centre of model base. As to displacement of alveolar bone in model A, $-1.1 \times 1.0^{-3} \sim 0.9 \times 10^{-3} \mathrm{~mm}$ displacement were found at alveolar process, and $-0.2 \times 1.0^{-3} \sim 0.0 \mathrm{~mm}$ displacement were found near alveolar base. With respect to displacement of PDL in model $\mathrm{B},-0.9 \times 1.0^{-3} \sim-0.2 \times 1.0^{-3} \mathrm{~mm}$ displacement were found from cervical area to root apex.

\subsection{Displacement Vector}

In model A (Fig. 2c), displacement vectors of both alveolar bone and crown-tooth root drew the same circular arc 


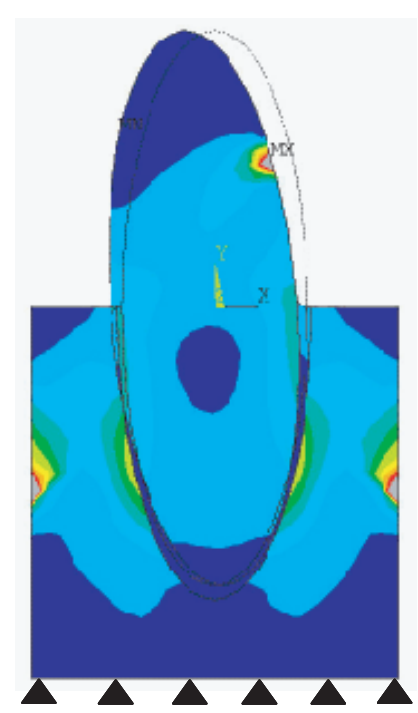

a

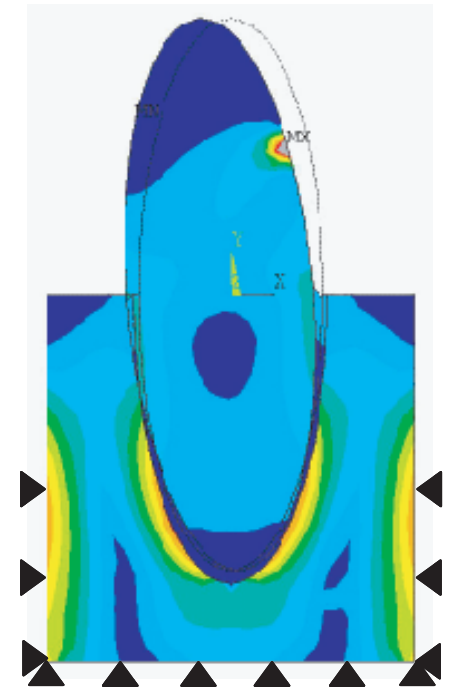

b

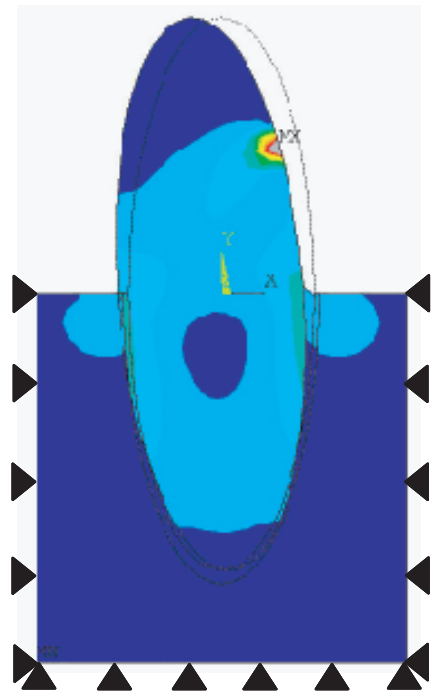

c

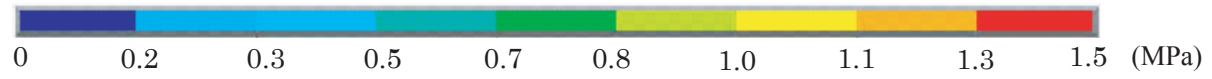

Fig. (4). Difference of equivalent (Von Mises) stress distribution on PDL models with various restraint conditions. a: Model B (base restrained), b: Model C (base + side 1/2restrained), c: Model D (a whole restrained).

centring on the model base. Tooth with PDL were deformed obliquely as one body. In model B (Fig. 3c), on the other hand, displacement vector of alveolar bone surrounded the PDL, whereas displacement vector of crown-tooth root drew circle centring on the middle of the root, making a turn due to viscoelastic characteristics of PDL.

\section{Analysis 2}

The behaviour of mandibular central incisor with PDL and various restraint sites.

\subsection{Equivalent Stress Distribution Pattern}

Fig. (4) demonstrates equivalent stress distribution patterns under various restraint conditions, such as model B (Fig. 4a), model C (Fig. 4b) and model D (Fig. 4c). No significant differences in stress distribution between the models were found in the cervical area of the tooth and PDL.

In alveolar bone of model $\mathrm{B}$ and model $\mathrm{C}$, relatively high stresses of $0.3 \sim 1.0 \mathrm{MPa}$ were observed at both sides of alveolar bone and around the root. Particularly model $\mathrm{C}$ showed significantly higher stress values compared with model B, e.g. $0.5 \sim 1.3 \mathrm{MPa}$ stresses were found around the area of the root.

In alveolar bone of model D, 0.3 MPa stresses generated in cervical margin of alveolar process were gradually decreased toward alveolar base.

\subsection{Displacement Distribution Pattern}

Fig. (5) shows displacement distribution pattern under various restraint conditions, such as model B (Fig. 5a), model C (Fig. 5b), and model D (Fig. 5c). No significant differences in displacement were found in tooth and PDL under any restraint conditions. In alveolar bone, however, displacement decreased toward alveolar base in accordance with expanding restraint area. Displacement distribution pattern under three different restraint conditions showed almost symmetric line against longitudinal axis of the tooth. Tooth and PDL were inclined from labial side to lingual side at the middle of the root as a border line.

\subsection{Displacement Vector}

Fig. (6) shows displacement vector of model B (Fig. 6a), model C (Fig. 6b), and model D (Fig. 6c), respectively. Under any restraint conditions, displacement vector of toothPDL continuum drew circle centring on the middle of roots. In alveolar bone, model $\mathrm{B}$ showed $\mathrm{M}$-shaped orientation of displacement vector surrounding PDL in alveolar bone. Model C drew circles centring on the root apex and on $1 / 2$ side of restraint sites, while model D drew circular arcs centring on the middle of the root.

\section{DISCUSSION}

\section{FE Method and Modelling}

Although actual measurements and clinical trials have been carried out to investigate the influence of the presence of PDL on tooth and alveolar bone behaviour, it is difficult to compare the test results [16-20], because tooth differs from each other in terms of geometry, morphology and dimensions. The FE method has become one of the most frequently used analysing methods when evaluating the dental related issues. Unlike with many other FE applications, certain assumptions need to be made when dealing with complicated set of teeth, bone or PDL. The FE method approach requires not only an agreement of dimensions and material properties of the experimental structures, but also precise calculation of the inner stresses. This makes the construction 


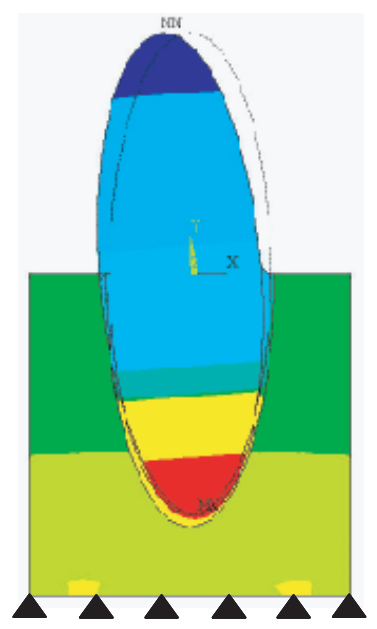

a

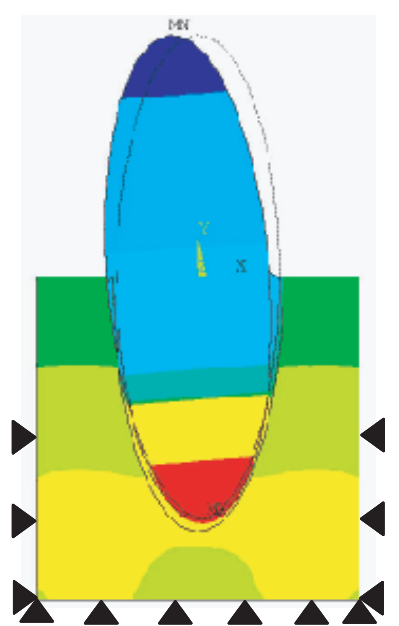

b

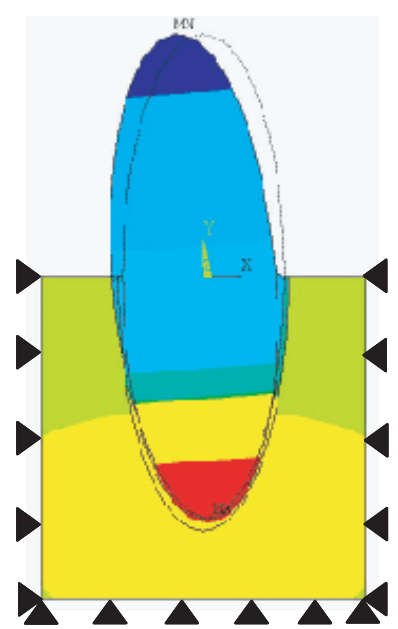

c

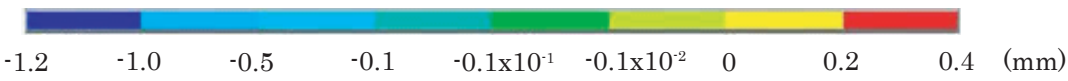

Fig. (5). Difference of displacement on PDL models with various restraint conditions.

a: Model B (base restrained), b: Model C (base + side 1/2 restrained), c: Model D (a whole restrained).

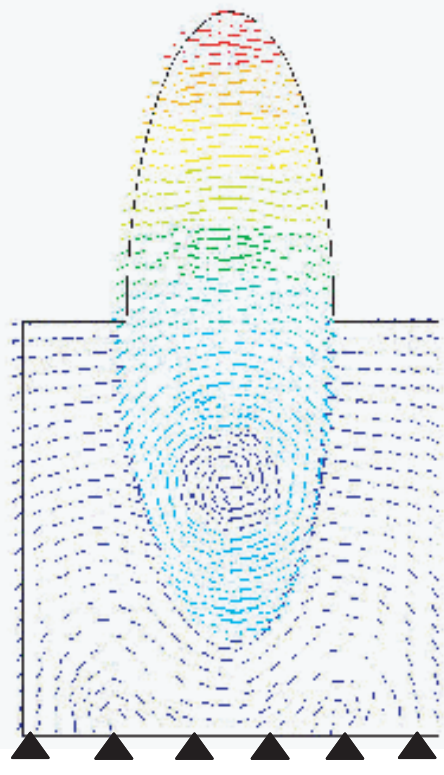

a

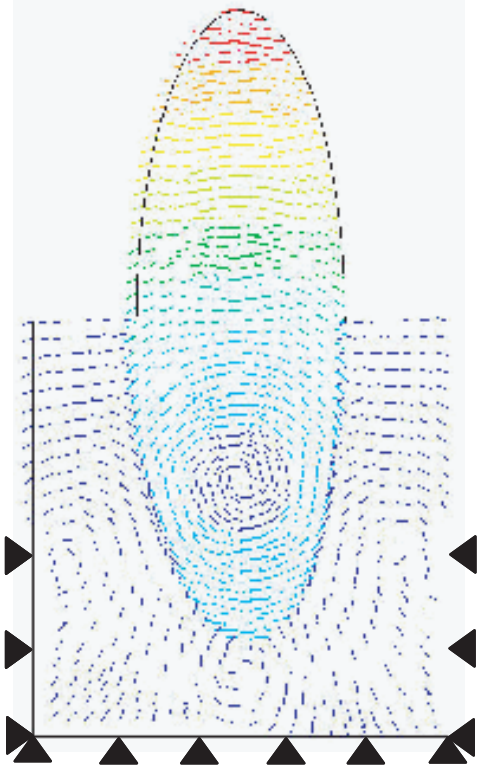

$\mathrm{b}$

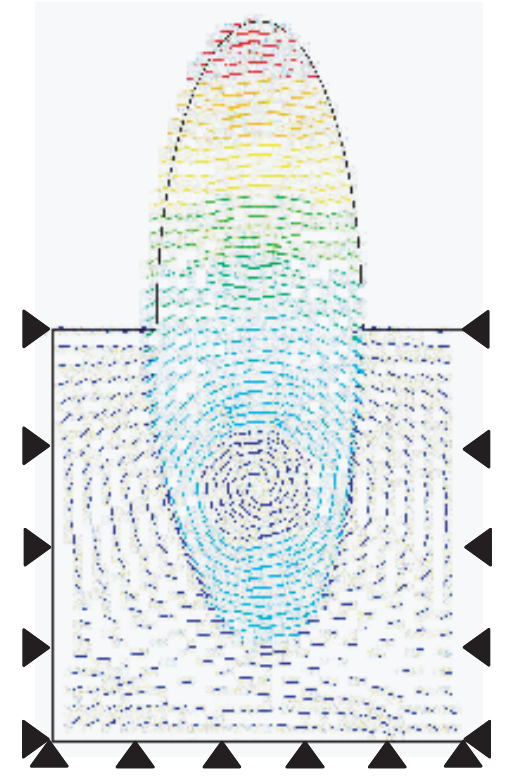

c

Fig. (6). Difference of vector indication of displacement on PDL models with various restraint conditions. a: Model B (base restrained), b: Model C (base + side 1/2 restrained), c: Model D (a whole restrained).

of FE models difficult. FE study was chosen for this study, because FE models remained same without being affected by the restraint sites. Furthermore, FE analysis enabled to evaluate the influence of different restraint sites [21-24].

In this study, a 2D FE model was chosen primarily because it enabled the analysis of stress, displacement and vector direction distribution, with different boundary conditions in the same FE model with identical material properties. If other variables would have been added, the simulated situation would have not identified the results of the stress distribution. In the previous study, 2D FE analysing method was used to evaluate the stress of the bone and implant [2527]. Evaluation of the stress distribution using a 2D FE model has showed as a very effective analyzing method in the dental science. The results of this study helped the selection of the boundary condition. However, within the limitation of this 2D FE model, the mechanical behaviour of the PDL and the remodelled bone remained naturally unknown, 
which should be taken into consideration upon the accurate clinical condition.

\section{The Effect of the Presence of PDL on Tooth Behaviour}

PDL plays an important role in the orthodontic tooth movement. A 2-D FE analysis was performed to clarify the influence of the presence of PDL on tooth behaviour [1]. No significant differences in equivalent stress induced in cervical area of the tooth were found between model A and model B. Model A, however, exhibited two times as high as stress values at cervical margin of alveolar process compared with model B. In model B, relatively high stress values were observed within alveolar bone around the root. This may be explained by the fact that PDL has the capacity to absorb stress and to transmit much reduced stress to alveolar bone.

Significant differences in displacement were found between model A (Fig. 2b) and model B (Fig. 3b). Model A demonstrated relatively small displacement compared with model B, and also exhibited deformed displacement distribution pattern around cervical area of alveolar bone. Model A was inclined to lingual direction pivoting on the centre of model base, whereas model B was inclined to lingual direction pivoting on the middle of the root as the centre of resistance, which might be influenced by lower Young's modulus PDL possesses. Since the model A assumed to have a perfect adherence (tooth and PDL, as well as PDL and alveolar bone), model A was thought to exhibit the same mechanical behaviour as a cantilever bridge, acting the fixed point as fulcrum. In short, restrained model base functioned as the centre of displacement. A bit of free tooth displacement within alveolar bone was allowed by including viscoelastic interference material with lower Young's modulus between the loading point and the fixed point [28, 29], thus enabling the centre of displacement to move from the fixed point to the middle of the root.

When simulating orthodontic tooth movement, it is essential to include PDL in FE models so that the stress distribution within tooth and alveolar bone are reduced, and orthodontic force necessary to move tooth are transmitted correctly. In addition, FE model with PDL made it possible to demonstrate the centre of resistance within a tooth root [29-34].

\section{The Effect of Various Restraint Sites on Tooth Behav- iour}

In order to better simulate the tooth displacement of natural oral environment, the evaluation of the influence of various restraint sites included PDL on tooth behaviour is critical. The present study was therefore done under three different restraint conditions.

When the effect of various restraint sites on equivalent stress distribution pattern was investigated, significant differences were observed within the alveolar bone. In model D (Fig. 4c), due to the influence of side restrained, stress generated within alveolar bone had a tendency to exhibit smaller value as it should be. In model C (Fig. 4b), due to the influence of lower $1 / 2$ alveolar bone restrained, applied force was not fully transmitted to areas around the root, as well as excessively high stress values were obtained at the specific sites of both sides of alveolar bone. In model B (Fig. 4a), applied force was fully transmitted within alveolar bone to areas around the root without being affected by base restraint. No significant differences in equivalent stress distribution pattern were found in tooth and PDL under any restraint conditions. In displacement, no significant differences were found between the models investigated, and all models were inclined from negative direction to positive direction (Fig. 5). Fig. (6) represents the result of displacement vector under three different restraint conditions, which revealed that all three models had the centre of resistance at the same position.

The results obtained so far revealed that various restraint sites did not significantly affect tooth and PDL, but had a significant effect on alveolar bone. Therefore it is advisable to choose a restraint site having no specific influence on stress distribution of alveolar bone, whenever evaluation of alveolar bone is crucial. The results of this study clarified that base restraint showed the best simulation of what actually occurs in oral environment.

\section{Clinical Implications}

The results of this study indicate that, during initial movement of PDL deformation, the presence of PDL enabled to alleviate excessive stress concentration generated in tooth and alveolar bone, to appropriately transmit orthodontic force necessary to move tooth. Additionally, the presence of PDL allowed the middle of the root to occur the centre of resistance for oblique inclination. Various restraint sites did not significantly affect tooth and PDL, but had a significant effect on alveolar bone, indicating that findings obtained in this study will be important guidelines to simulate orthodontic tooth movement.

For the orthodontist, clinicians must know the mechanical behaviour of tooth, PDL and bone under the orthodontic force to prevent the root resorption. Now these days, new method using the micro implant technique for anchorage of the orthodontic force is available. There are no PDL around the micro implant. This technique will make more complicate the mechanical behaviour of tooth movement, because tooth with PDL and micro implant (without PDL) are connected and pull each other. This manuscript will help the orthodontists to understand about this kind of mechanical behaviour.

\section{CONCLUSION}

In the present study, 2D FE models were constructed to simulate mechanical behaviour observed during the initial movement of PDL deformation, and evaluated the effects of the presence of PDL and various restraint sites on tooth behaviour, stress distribution pattern and displacement. Within the limitation of this in vitro study, the following conclusions were drawn:

1. By incorporating PDL in FE models, excessively large stress values and deformation generated in a tooth and alveolar bone were relieved, and orthodontic force necessary for tooth displacement was transmitted correctly. Furthermore, the presence of PDL enabled to make the centre of resistance for oblique inclination at the middle of the root. 
2. Since various restraint conditions had a significant effect on alveolar bone, the least affected site of base restraint should be chosen when evaluation of alveolar bone is critical.

\section{REFERENCES}

[1] Graber T M, Swain BF. Orthodontics current principles and techniques. $1^{\text {st }}$ ed. St. Louis Mosby Inc. 1985; pp.13-128.

[2] Damon DH. The Damon low-friction bracket: a biologically compatible straight-wire system. J Clin Orthod 1998; 32: 670-80.

[3] Kapur R, Sinha PK, Nanda RS. Frictional resistance of the Damon SL bracket. J Clin Orthod 1998; 32: 485-9.

[4] Thorstenson GA. Kusy RP. Resistance to sliding of self- ligating brackets versus conventional stainless steel twin brackets with second-order angulation in the dry and wet (saliva) states. Am J Orthod Dentofacial Orthop 2001; 120: 361-70.

[5] Berger JL. The influence of the SPEED bracket's self-ligating design on force levels in tooth movement: a comparative in vitro study. Am J Orthod Dentofacial Orthop 1990; 97: 219-28.

[6] Sims AP, Waters NE, Birnie DJ, Pethybridge RJ. A comparison of the forces required to produce tooth movement in vitro using two self-ligating brackets and a pre-adjusted bracket employing two types of ligation. Eur J Orthod 1993; 15: 377-85.

[7] Kojima Y, Takano M, Fukui H, Mizutani N, Hasegawa J. A simple method for calculating the initial tooth mobility and stress distribution in the periodontal ligament. Jpn J Dent Mater 1999; 18: 21016.

[8] Hioki M, Shinya A, Nakahara R, Vallittu PK, Nakasone Y, Shinya A. Shear bond strength and FEM of a resin-modified glass ionomer cement-effects of tooth enamel shape and orthodontic bracket base configuration. Dent Mater J 2007; 26: 700-7.

[9] Shinya A, Lassila LVJ, Vallittu PK. The effect of preparation design on the marginal stress of resin-bonded metal-free crowns: a finite element study. Int J Prosthodont 2008; 21: 151-3.

[10] Ootaki M, Shinya A, Gomi H, Shinya A, Nakasone Y. Optimum design for fixed partial dentures made of hybrid resin with glass fiber reinforcement by finite element analysis: effect of vertical reinforced thickness on fiber frame. Dent Mater J 2007; 26: 280-9.

[11] Nakamura T, Ohyama T, Waki T, et al. Finite element analysis of fiber-reinforced fixed partial dentures. Dent Mater J 2005; 24: 2759.

[12] Baek SH, Shin SJ, Ahn SJ, Chang YI. Initial effect of multiloop edgewise archwire on the mandibular dentition in class III malocclusion subjects. A three-dimensional finite element study. Eur $\mathbf{J}$ Orthod 2008; 30: 10-5.

[13] Veziroglu F, Uckan S, Ozden UA, Arman A. Stability of zygomatic plate-screw orthodontic anchorage system. Angle Orthod 2008; 78: 902-7.

[14] Wheeler RC. Dental Anatomy, Physiology and Occlusion, $5^{\text {th }}$ ed. Philadelphia: Saunders 1974; pp. 229-36.

[15] Otsubo J. A study on the tooth material in Japanese adults of normal occlusion, its relationship to coronal and basal arches. J Jpn Orthod Soc 1957; 16: 36-46.

[16] Cardaropoli G, Araújo M, Hayacibara R, Sukekava F, Lindhe J. Healing of extraction sockets and surgically produced-augmented and non-augmented - defects in the alveolar ridge. An experimental study in the dog. J Clin Periodontol 2005; 32: 435-40.
[17] Rygh P, Bowling K, Hovlandsdal L, Williams S. Activation of the vascular system: a main mediator of periodontal fiber remodeling in orthodontic tooth movement. Am J Orthod Dentofacial Orthop 1986; 89: 453-68.

[18] Verna C, Zaffe D, Siciliani G. Histomorphometric study of bone reactions during orthodontic tooth movement in rats. Bone 1999; 24: 371-9.

[19] Ong CK, Walsh LJ, Harbrow D, Taverne AA, Symons AL. Orthodontic tooth movement in the prednisolone-treated rat. Angle Orthod 2000; 70: 118-25.

[20] Kojima Y, Fukui H, Hasegawa J. A mechanical analysis for distal tipping movement of lower second molar. Jpn J Dent Mater 2001; 20: 32-9.

[21] Ona M, Wakabayashi N. Influence of alveolar support on stress in periodontal structures. J Dent Res 2006; 85: 1087-91.

[22] Cattaneo PM, Dalstra M, Melsen B. The finite element method: a tool to study orthodontic tooth movement. J Dent Res 2005; 84: 428-33.

[23] Qian H, Chen J, Katona TR. The influence of PDL principal fibers in a 3-dimensional analysis of orthodontic tooth movement. Am J Orthod Dentofacial Orthop 2001; 120: 272-9.

[24] Proffit WR. Contemporary Orthodontics. 3rd ed. St. Louis Mosby Inc. 2000; pp. 340-44.

[25] Quaresma SE, Cury PR, Sendyk WR, Sendyk C. A finite element analysis of two different dental implants: stress distribution in the prosthesis, abutment, implant, and supporting bone. J Oral Implantol 2008; 34: 1-6.

[26] Ozçelik T, Ersoy AE. An investigation of tooth/implant-supported fixed prosthesis designs with two different stress analysis methods: an in vitro study. J Prosthodont 2007; 16: 107-16.

[27] Hedia HS. Design of functionally graded dental implant in the presence of cancellous bone. J Biomed Mater Res B Appl Biomater 2005; 75: 74-80.

[28] Shimizu Y, Usui K, Araki K, Kurosaki N, Takanobu H, Takanishi A. Study of finite element modeling from CT images. Dent Mater J 2005; 24: 447-55.

[29] Kitagawa T, Tanimoto Y, Nemoto K, Aida M. Influence of cortical bone quality on stress distribution in bone around dental implant. Dent Mater J 2005; 24: 219-24.

[30] Watanabe F, Hata Y, Komatsu S, Ramos TC, Fukuda H. Finite element analysis of the influence of implant inclination,loading position, and load direction on stress distribution. Odontology 2003; 91: 31-6.

[31] Kojima Y, Fukui H. Calculation of natural frequencies of teeth supported with the periodontal ligament. Dent Mater J 2007; 26: 254-9.

[32] Jafari A, Shetty S, Kumar M. Study of stress distribution and displacement of various craniofacial structures following application of transverse orthopedic forces. A three-dimensional FEM study. Angle Orthod 2003; 73: 12-20.

[33] Chen F, Terada K, Handa K. Anchorage effect of various shape palatal osseointegrated implants: A finite element study. Angle Orthod 2005; 75: 378-85.

[34] Natali AN, Pavan PG, Scarpa C. Numerical analysis of tooth mobility: formulation of a non-linear constitutive law for the periodontal ligament. Dent Mater 2004; 20: 623-9. 